\title{
Tracheal Mucositis, CTCAE
}

National Cancer Institute

\section{Source}

National Cancer Institute. Tracheal Mucositis, CT CAE. NCI Thesaurus. Code C143881.

A disorder characterized by an inflammation or ulceration involving the mucous membrane of the trachea. 